\title{
Incidence Risk and Independent Predictors of Prolonged Air Leak in 269 Consecutive Pulmonary Resection Patients over Nine Months: A Single-Center Retrospective Cohort Study
}

\author{
Connie Drewbrook¹, Samarpita Das', Dorsa Mousadoust², Basil Nasir ${ }^{1,2}$, John Yee ${ }^{1,2}$, Anna McGuire ${ }^{1,2}$ \\ ${ }^{1}$ Faculty of Medicine, University of British Columbia, Vancouver, Canada \\ ${ }^{2}$ Department of Surgery, Division of Thoracic Surgery, University of British Columbia, Vancouver, Canada \\ Email: anna.mcguire@vch.ca, conndrew@gmail.com, shyamadas09@gmail.com, dorsamousadoust@alumni.ubc.ca, john.yee@vch.ca, \\ basil.nasir@vch.ca
}

How to cite this paper: Drewbrook, C., Das, S., Mousadoust, D., Nasir, B., Yee, J. and McGuire, A. (2016) Incidence Risk and Independent Predictors of Prolonged Air Leak in 269 Consecutive Pulmonary Resection Patients over Nine Months: A SingleCenter Retrospective Cohort Study. Open Journal of Thoracic Surgery, 6, 33-46. http://dx.doi.org/10.4236/ojts.2016.64006

Received: October 12, 2016

Accepted: November 6, 2016

Published: November 9, 2016

Copyright $\odot 2016$ by authors and Scientific Research Publishing Inc. This work is licensed under the Creative Commons Attribution International License (CC BY 4.0).

http://creativecommons.org/licenses/by/4.0/

\section{Abstract}

Introduction: Prolonged air leak (PAL) is a common complication following pulmonary resection. It is associated with pneumonia, empyema, increased length of hospital stay and health-care costs. Intraoperative techniques have been developed to mitigate the risk of developing a PAL, but for their use to be efficient, identification of patients at risk for PAL is necessary. Aim: To determine the incidence of PAL following lobectomy and lesser pulmonary resections, risk factors for development of PALs, and the impact of PAL on hospital stay and readmission rates. Methods: The following variables were analyzed as PAL risk factors: patient characteristics of age, sex, body mass index (BMI), forced minute expiratory volume and capacity ratio (FEV1 and FEV1/FVC), diffusion capacity (DCLO), and transplant recipient status. Validated scoring systems included the Charlson Comorbidity Index (CCI), Medical Research Council (MRC) dyspnea score, and Eastern Cooperative Oncology Group (EGOC) score. Surgical factors included surgical technique, unplanned conversion from video-assisted thoracoscopic surgery (VATS) to thoracotomy, location and extent of resection, presence of adhesions, completeness of fissures, and method of fissure completion. Length of hospital stay and readmission rates were analyzed. Statistical tests performed on the data include univariate and multivariate logistic regression analyses. Results: Over the 9-month study duration there were 269 lung resections, of which 31 (11.52\%) had an air leak lasting longer than 5 days. Mean length of stay in hospital was significantly longer in patients with PAL compared to the control group (13 vs 5 days, $P<0.001$ ). Significant risk factors for PAL from multivariate analysis include normal BMI $(P=0.009)$, right upper lobectomy $(P=0.001)$, and un- 
planned conversion from video-assisted thoracoscopic surgery (VATS) to thoracotomy $(P=0.023)$. Conclusion: The incidence of PAL in our study population is similar to that found in previous studies. PAL prolongs hospital length of stay. Normal BMI, right upper lobectomy, and unplanned conversion from VATS to thoracotomy are risk factors for PAL.

\section{Keywords}

Pulmonary Resection, Air Leak, Risk Factors, Length of Stay, Thoracic Surgery

\section{Introduction}

Air leak following pulmonary resection is a common problem and in most cases is due to an alveolar-pleural fistula [1]. The definition of a prolonged air leak (PAL) varies in the literature, but the currently accepted definition is an air leak lasting 5 or more days postoperatively [2] which is the average length of stay (LOS) in hospital following lobectomy [3]. In previous studies, PAL has been found to occur in up to $26 \%$ of patients following lobectomy and lesser pulmonary resections [4] [5] [6] [7] [8].

The most consistent risk factors for PAL in the literature are related to reduced pulmonary function due to chronic obstructive pulmonary disease [5] [8] [9] [10] [11] [12]. Additional risk factors that have been described include reduced diffusing capacity [9] [12], upper resections or bilobectomy [5], pleural adhesions [5] [12], relatively lower BMI [13] [14], increased dyspnea score [14], concurrent pneumothorax [9] [10], lobectomy [10] [14], preoperative inhaled or systemic steroid use [10] [15], radiologic findings of severe emphysema [8], and histopathologic findings of emphysema [8].

In most cases PAL is self-limited [10], but it is associated with increased postoperative morbidity through increased rates of pulmonary and pleural complications such as empyema, pneumonia, and atelectasis [5] [8] [9]. Furthermore, the impact on the healthcare system is significant as it prolongs hospital stay by 4 to 13 days [8] [9] [16] and as a result increases health-care costs [17].

Due to the clinical implications of PAL, intraoperative methods have been developed to mitigate this risk and include pleural tenting, pneumoperitoneum, staple-line buttressing, and surgical sealants [18]. These techniques are not recommended to be performed routinely as not all patients are expected to benefit from their use [19]. Additionally, they are more time consuming and costly [19]. In order to identify patients at increased risk of PAL who may benefit from the use of intraoperative preventative techniques, several studies have identified risk factors for PAL and devised scoring systems to risk stratify patients [12] [13] [14].

The aim of this retrospective cohort study is to investigate the incidence of PAL following pulmonary resection and the most common risk factors for PAL in this group of patients. Risk factor categories include patient characteristics, validated scoring methods, and surgical factors. The Charlson Comorbidity Index (CCI) was developed as a 
weighted index of comorbid disease [20] and has been found to be a strong predictor of major complications following surgery for patients with non-small cell lung cancer [21]. We plan to investigate whether the CCI can be used as a predictor for PAL following resection.

\section{Materials and Methods}

All lobectomies and lesser resections performed at Vancouver General Hospital from October 2015 to July 2016 were included in the study. Inclusion criteria includes inpatients on the thoracic surgery ward, aged 18 and older, who have undergone pulmonary resection for benign or malignant causes. Patients who underwent pneumonectomy were excluded. Ethics approval was provided by the UBC Clinical Research Ethics Board.

Prospectively collected patient information was retrospectively obtained from the electronic medical record and hospital chart, and consisted of a patient's history and physical examination, MRC dyspnea score [22], ECOG score [23], pulmonary function tests, operation report, and pathology report.

Patient information collected included sex, age, BMI, Charlson Comorbidities, smoking status, alcoholism, FEV and FVC volumes and percent predicted, FEV/FVC ratio, diffusing capacity (DLCO), location and type of resection, presence of adhesions, completeness of fissures, method of completion for incomplete fissures, histology, TNM staging for non-small cell lung cancers, and primary diagnosis. The CCI is calculated as a weighted sum of 19 retrospectively collected patient comorbid conditions [20]. There were no patients with hemiplegia, moderate to severe liver disease, or AIDS, so these categories were not included in the analysis.

Pulmonary resections were performed by one of three thoracic surgeons at Vancouver General Hospital by video-assisted thoracoscopic surgery (VATS), thoracotomy, or midline sternotomy. Incomplete fissures were mainly completed with a stapler, although in a minority of cases electrocautery or a combination of electrocautery and staples were used.

One or two chest tubes were inserted and connected to a Pleur-Evac (Teleflex Medical, Wayne, PA) or Topaz (Medela, Baar, Switzerland) digital drainage device. The definition used for PAL was an air leak lasting 5 or more days.

Statistical analysis was performed using Stata 12 statistical software. Categorical variables were analyzed using the chi square test or Fisher exact test, whereas, continuous variables were analyzed using the two-tailed t-test. Univariate and multivariate analyses were performed. A $P$-value of $<0.05$ was considered significant.

\section{Results}

\subsection{Patient Characteristics}

Over the 9-month duration of the study there were 269 lung resections that met inclusion criteria. There were 135 men (50.2\%) and 134 (49.8\%) women with a mean age of 61.35 years, as is shown in Table 1 . 
Table 1. Indications for pulmonary resection of 269 consecutive pulmonary resections, October $1^{\text {st }} 2015$ to June $30^{\text {th }} 2016$, by prolonged air leak status.

\begin{tabular}{|c|c|c|c|c|}
\hline Primary diagnosis & $\begin{array}{c}\text { Total } \\
\mathrm{n}=269 \\
(\text { No., \%) }\end{array}$ & $\begin{array}{l}\text { Control group } \\
\text { air leak }<5 \text { days } \\
\mathrm{n}=238(\text { No., } \%)\end{array}$ & $\begin{array}{c}\text { Air leak } \geq 5 \text { days } \\
\mathrm{n}=31 \text { (No., } \%)\end{array}$ & $P$-value ${ }^{*}$ \\
\hline Lung cancer & $165(61.34)$ & $141(59.24)$ & $24(77.42)$ & \\
\hline Lung metastases & $31(11.52)$ & $31(13.03)$ & 0 & \\
\hline Recurrent pneumothorax & $25(9.29)$ & $22(9.24)$ & $3(9.68)$ & 0.056 \\
\hline ILD & $18(6.69)$ & $18(7.56)$ & 0 & \\
\hline Other (Benign lung lesions, infection) & $30(11.15)$ & $26(10.92)$ & $4(12.90)$ & \\
\hline
\end{tabular}

${ }^{\star}$ Chi square test of association for categorical variables; ${ }^{*}$ Two tail T-test of association for continuous variables.

PAL occurred in 31 patients (11.52\%) and was most frequently observed in patients undergoing resection for primary lung cancer, followed by other causes and pneumothorax, as is shown in Table 2.

Patients with a normal BMI were more likely to have PAL compared to those with an overweight BMI $(P=0.007)$ on univariate analysis. For those with PAL there was a trend for increased age $(P=0.132)$. The proportion of PAL was highest in the $70-79$ years age group $(n=12)$, but this did not reach statistical significance $(P=0.32)$. There is a trend for association of PAL with age $\geq 70$ years $(P=0.105)$. Non-significant patient characteristics include mean age $(P=0.13)$, sex $(P=0.83)$, FEV1\% predicted $(P=$ $0.89)$, FEV1/FVC\% $(P=0.38)$, DLCO $\%(P=0.38)$.

\subsection{Validated Scoring Methods}

When ECOG status is grouped into two categories: $0-1$ and $\geq 2$, there is very weak evidence of association of ECOG $\geq 2$ with PAL $(P=0.099)$. Statistically insignificant validated scores include mean CCI $(P=0.38)$ and MRC dyspnea score $(P=0.55)$, as is shown in Table 3.

\subsection{Current Habits and Charlson Comorbidities}

Cerebrovascular disease was found to be associated with PAL $(P=0.030)$ and there was a trend for association of uncomplicated diabetes with PAL $(P=0.145)$. All remaining comorbidities were not found to be significantly associated with PAL, as is shown in Table 4.

\subsection{Length of Stay and Readmission Outcomes}

LOS was significantly longer for patients in the air leak group with a mean length of stay of 12.61 days compared to 4.95 days in the control group $(P<0.001)$. PAL was not significantly associated with increased readmission rates. Readmission at 30 days was $3.2 \%(n=1)$ in the PAL group and $0.42 \%(n=1)$ in the control group $(P=0.22)$. Readmission at 90 days was 3.2\% $(n=1)$ in the PAL group and $2.1 \%(n=5)$ in the control group $(P=0.5)$. Frequency of readmission at 30 and 90 days was small and similar between groups. There were 2 deaths in the study, both in the control group, and due to 
Table 2. Univariate analysis of patient characteristics for 269 consecutive pulmonary resections, October $1^{\text {st }} 2015$ to June $30^{\text {th }} 2016$, by prolonged air leak status.

\begin{tabular}{|c|c|c|c|c|}
\hline Variable & $\begin{array}{c}\text { Total } \\
(\text { No., \%) }\end{array}$ & $\begin{array}{l}\text { Control group } \\
\text { air leak }<5 \text { days } \\
(\text { No., } \%)\end{array}$ & $\begin{array}{c}\text { Air leak } \geq 5 \text { days } \\
(\text { No., } \%)\end{array}$ & $P$-value ${ }^{*}$ \\
\hline All pulmonary resections & $269(100)$ & $238(88.48)$ & $31(11.52)$ & - \\
\hline \multicolumn{5}{|l|}{ Age } \\
\hline Age: Mean (SD) & $61.35(15.8,59.5-63.3)$ & $60.83(16.1)$ & $65.39(12.49)$ & 0.132 \\
\hline \multicolumn{5}{|l|}{ Age category (years): } \\
\hline$<50$ & 43 (15.99) & 39 (16.39) & $4(12.90)$ & \multirow{5}{*}{0.322} \\
\hline $51-59$ & $59(21.93)$ & $52(21.85)$ & $7(22.58)$ & \\
\hline $60-69$ & $80(29.74)$ & $74(31.09)$ & $6(19.35)$ & \\
\hline $70-79$ & $65(24.16)$ & $53(22.27)$ & $12(38.71)$ & \\
\hline$>80$ & $22(8.18)$ & $20(8.40)$ & $2(6.45)$ & \\
\hline Age $\geq 70$ years & $87(32.34)$ & $73(30.67)$ & $14(45.16)$ & 0.105 \\
\hline \multicolumn{5}{|l|}{ Sex: } \\
\hline Male & $135(50.19)$ & $120(50.42)$ & $15(48.39)$ & \multirow{2}{*}{0.831} \\
\hline Female & $134(49.81)$ & $118(49.58)$ & $16(51.61)$ & \\
\hline \multicolumn{5}{|l|}{ BMI } \\
\hline \multicolumn{5}{|l|}{ BMI: $(\mathrm{n}=243)$} \\
\hline Mean (SD) & $25.59(5.86)$ & $25.77(6.05)$ & $25.59(3.95)$ & 0.1733 \\
\hline \multicolumn{5}{|l|}{ BMI category: $(\mathrm{n}=243)$} \\
\hline Underweight $(<18.5)$ & $18(7.41)$ & $18(8.41)$ & $0(0)$ & \multirow{3}{*}{$\begin{array}{c}0.007 \\
\text { (Fisher exact) }\end{array}$} \\
\hline Normal (18.5 - 25) & $110(45.27)$ & $89(41.59)$ & $21(72.41)$ & \\
\hline Overweight (>25) & $115(47.33)$ & $107(50.00)$ & $8(27.59)$ & \\
\hline \multicolumn{5}{|c|}{ Pulmonary function testing } \\
\hline \multicolumn{5}{|l|}{ FEV1\%: $(\mathrm{n}=210)$} \\
\hline Mean (SD) & $86.27(8.46)$ & $86.33(18.50)$ & $85.8(18.56)$ & 0.8923 \\
\hline \multicolumn{5}{|l|}{ FEV1 category $(\mathrm{n}=210)$} \\
\hline$<60 \%$ & $15(7.14)$ & $12(6.49)$ & $3(12.00)$ & \multirow{3}{*}{0.480} \\
\hline $60 \%-80 \%$ & $66(31.43)$ & $60(32.43)$ & $6(24.00)$ & \\
\hline$>80 \%$ & $129(61.43)$ & $113(61.08)$ & $16(64.00)$ & \\
\hline FEV $1<80 \%$ & $81(38.57)$ & $72(38.92)$ & $9(36.00)$ & 0.778 \\
\hline FEV $1<60 \%$ & $15(7.14)$ & $12(6.49)$ & $3(12.00)$ & 0.397 \\
\hline \multicolumn{5}{|l|}{ FEV1/FVC\% $(\mathrm{n}=206)$} \\
\hline Mean (SD) & $72.80(11.89)$ & $73.08(11.73)$ & $70.90(13.0)$ & 0.3843 \\
\hline \multicolumn{5}{|l|}{ FEV1/FVC\% category } \\
\hline$<60 \%$ & $23(11.17)$ & $20(11.11)$ & $3(11.54)$ & \multirow{3}{*}{0.716} \\
\hline $60 \%-80 \%$ & $130(63.11)$ & $112(62.22)$ & $18(69.23)$ & \\
\hline$>80 \%$ & $53(25.73)$ & $48(26.67)$ & $5(19.23)$ & \\
\hline \multicolumn{5}{|l|}{ DLCO $\%(n=202)$} \\
\hline Mean (SD) & $76.05(18.31)$ & $76.45(18.23)$ & $72.91(19.03)$ & 0.3841 \\
\hline \multicolumn{5}{|l|}{ DLCO \% category } \\
\hline$<60 \%$ & $36(17.82)$ & $30(16.76)$ & $6(26.09)$ & \multirow{3}{*}{0.353} \\
\hline $60 \%-80 \%$ & $79(39.11)$ & $69(38.55)$ & $10(43.48)$ & \\
\hline$>80 \%$ & $79(43.07)$ & $80(44.69)$ & $7(30.43)$ & \\
\hline
\end{tabular}


Table 3. Validated scoring tools for study population of 269 consecutive pulmonary resections, October $1^{\text {st }} 2015$ to June $30^{\text {th }} 2016$, by prolonged air leak status.

\begin{tabular}{|c|c|c|c|c|}
\hline Variable & $\begin{array}{c}\text { Total } \\
\text { (No., \%) }\end{array}$ & $\begin{array}{l}\text { Control group } \\
\text { air leak }<5 \text { days } \\
(\text { No., } \%)\end{array}$ & $\begin{array}{c}\text { Air leak } \geq 5 \text { days } \\
(\text { No., \%) }\end{array}$ & $P$-value ${ }^{*}$ \\
\hline \multicolumn{5}{|c|}{ CC Index $(n=269)$} \\
\hline Mean (SD) & $1.6(1.7)$ & $1.6(1.7)$ & $1.5(1.3)$ & 0.3786 \\
\hline \multicolumn{5}{|c|}{ CC index category: $(n=269)$} \\
\hline 0 & $86(31.97)$ & $77(2.35)$ & $9(29.03)$ & \multirow{4}{*}{0.300} \\
\hline $1-2$ & $126(46.84)$ & $110(46.22)$ & $16(51.61)$ & \\
\hline $3-4$ & $37(13.75)$ & $31(13.03)$ & $6(19.35)$ & \\
\hline$\geq 5$ & $20(7.43)$ & $20(8.40)$ & 0 & \\
\hline \multicolumn{5}{|c|}{$\begin{array}{l}\text { ECOG performance status } \\
(n=266) \text { category: }\end{array}$} \\
\hline ECOG 0 & $165(62.03)$ & $148(62.71)$ & $17(56.67)$ & \multirow{4}{*}{0.420} \\
\hline ECOG 1 & $84(31.58)$ & $75(31.78)$ & $9(30.0)$ & \\
\hline ECOG 2 & $12(4.51)$ & $9(3.81)$ & $3(10.0)$ & \\
\hline ECOG 3 & $5(1.88)$ & $4(1.69)$ & $1(1.88)$ & \\
\hline \multicolumn{5}{|c|}{ ECOG grouping: $(n=266)$} \\
\hline ECOG 0 - 1 & $249(93.61)$ & $223(94.49)$ & $26(86.67)$ & \multirow{2}{*}{0.099} \\
\hline $\mathrm{ECOG} \geq 2$ & $17(6.39)$ & $13(5.51)$ & $4(13.33)$ & \\
\hline \multicolumn{5}{|c|}{ MRC dyspnea scores $(n=266)$} \\
\hline 1 & $172(64.66)$ & $154(65.25)$ & $18(60.00)$ & \multirow{4}{*}{0.552} \\
\hline 2 & $73(27.44)$ & $64(27.12)$ & $9(30.00)$ & \\
\hline 3 & $15(5.64)$ & $12(5.08)$ & $3(10.00)$ & \\
\hline 4 & $6(2.26)$ & $6(2.54)$ & 0 & \\
\hline
\end{tabular}

Table 4. Current habits and Charlston comorbidities for study population of 269 consecutive pulmonary resections, October $1^{\text {st }} 2015$ to June $30^{\text {th }} 2016$, by prolonged air leak status.

\begin{tabular}{|c|c|c|c|c|}
\hline Variable & $\begin{array}{c}\text { Total } \\
\text { (No., \%) }\end{array}$ & $\begin{array}{l}\text { Control group } \\
\text { air leak }<5 \text { days } \\
\mathrm{n}=238 \text { (No., \%) }\end{array}$ & $\begin{array}{c}\text { Air leak } \geq 5 \text { days } \\
\mathrm{n}=31(\text { No., } \%)\end{array}$ & $P$-value ${ }^{*}$ \\
\hline \multicolumn{5}{|l|}{ Current habit } \\
\hline Smoker & $32(11.90)$ & $27(11.34)$ & $5(16.13)$ & 0.389 \\
\hline Alcoholism & $7(2.60)$ & $6(2.52)$ & $1(3.23)$ & 0.580 \\
\hline \multicolumn{5}{|l|}{ Comorbid disease } \\
\hline CAD & $28(10.41)$ & $25(10.50)$ & $3(9.68)$ & 0.887 \\
\hline $\mathrm{CHF}$ & $7(2.60)$ & $2(6.45)$ & $5(2.10)$ & 0.187 \\
\hline PVD & $11(4.09)$ & $2(6.45)$ & $9(3.78)$ & 0.369 \\
\hline Cerebrovascular disease & $24(8.92)$ & $18(7.56)$ & $6(19.35)$ & 0.030 \\
\hline Chronic lung disease & $96(35.69)$ & $13(41.94)$ & $83(34.87)$ & 0.434 \\
\hline Chronic cognitive deficit & $1(0.37)$ & $1(0.42)$ & 0 & 0.718 \\
\hline Connective tissue disease & $15(5.58)$ & $12(5.04)$ & $3(9.68)$ & 0.393 \\
\hline PUD & $6(2.23)$ & $6(2.52)$ & 0 & 0.371 \\
\hline DM uncomplicated & $32(11.90)$ & $31(13.03)$ & $1(3.23)$ & 0.145 \\
\hline DM complicated & $3(1.12)$ & $3(1.26)$ & 0 & 0.530 \\
\hline CKD moderate to severe & $6(2.23)$ & $5(2.10)$ & $1(3.23)$ & 0.524 \\
\hline Leukemia & $3(1.12)$ & $3(1.26)$ & 0 & 0.530 \\
\hline Lymphoma & $5(1.86)$ & $4(1.68)$ & $1(3.23)$ & 0.460 \\
\hline Solid tumour no mets & 45 & $39(16.39)$ & $6(19.35)$ & 0.617 \\
\hline Solid tumour with mets & $11(4.09)$ & $11(4.62)$ & 0 & 0.622 \\
\hline Liver disease mild & $7(2.60)$ & $7(2.94)$ & 0 & 0.333 \\
\hline HIV/AIDS & $1(0.37)$ & $1(0.42)$ & 0 & 0.718 \\
\hline
\end{tabular}


cardiac arrest $(n=1)$ and acute respiratory distress syndrome $(\operatorname{ARDS})(n=1)$, as is shown in Table 5.

\subsection{Surgical Factors}

The majority of surgeries (93.3\%) were performed using video-assisted thoracoscopic surgery (VATS). Surgical factors that are statistically significant risk factor for PAL include VATS that involved an unplanned conversion to thoracotomy $(P=0.011)$ and RUL lobectomy $(P=0.001)$. Any upper lobectomy, defined as right upper lobectomy, left upper lobectomy, or left apical trisegmentectomy, was significantly associated with $\operatorname{PAL}(P=0.014)$, as well as any upper lobectomy or bilobectomy $(P=0.012)$. Also significantly associated with PAL was the presence of adhesions $(P<0.001)$, as is shown in Table 6.

\subsection{Multivariate Analysis}

The results following multivariate regression analysis are shown in Table 7 and Table 8. Significant predictors of PAL following multivariate regression include unplanned conversion from VATS to thoracotomy $(P=0.023)$, right upper lobectomy $(P=0.001)$, and normal BMI compared to an overweight BMI $(P=0.009)$.

\section{Discussion}

The aim of this retrospective cohort study was to investigate the incidence of PAL following pulmonary resection and the most common risk factors for PAL in this group of patients. We defined a prolonged air leak as an air leak lasting 5 or more days postoperatively. In previous studies, PAL has been found to occur in up to $26 \%$ of patients following lobectomy and lesser pulmonary resections [4] [5] [6] [7] [8] and is associated with increased postoperative morbidity through increased rates of pulmonary and pleural complications such as empyema, pneumonia, and atelectasis [5] [8] [9]. In this study the incidence of prolonged air leak was $11.52 \%$ which is similar to the incidence found in previous studies.

Previous studies have found that the impact of PAL on the healthcare system is significant as it prolongs hospital stay by 4 to 13 days [8] [9] [16] and as a result increases

Table 5. Length of stay, mortality, and readmission univariable analysis for study population of 269 consecutive pulmonary resections, October $1^{\text {st }} 2015$ to June $30^{\text {th }} 2016$, by prolonged air leak status.

\begin{tabular}{lcccc}
\hline \multicolumn{1}{c}{ Variable } & $\begin{array}{c}\text { Total } \\
(\text { No., } \%)\end{array}$ & $\begin{array}{c}\text { Control group } \\
\text { air leak }<5 \text { days } \\
\mathbf{n}=238(\text { No., } \%)\end{array}$ & $\begin{array}{c}\text { Air leak } \geq 5 \text { days } \\
\mathbf{n}=\mathbf{3 1}(\text { No., \%) }\end{array}$ & $P$-value* \\
\hline LOS (days) $\mathrm{n}=$ 269 Mean (SD) & $5.83(5.47)$ & $4.95(4.65)$ & $12.61(6.54)$ & $<0.001$ \\
All cause 30 day mortality & $2(0.74)$ & $2(0.84)$ & 0 & 0.608 \\
30 day readmit & $2(0.74)$ & $1(0.42)$ & $1(3.23)$ & 0.218 \\
90 day readmit & $6(2.23)$ & $5(2.10)$ & $1(3.23)$ & 0.524 \\
\hline
\end{tabular}


Table 6. Surgical risk factors for study population of 269 consecutive pulmonary resections, October $1^{\text {st }} 2015$ to June $30^{\text {th }} 2016$, by prolonged air leak status.

\begin{tabular}{|c|c|c|c|c|}
\hline Variable & $\begin{array}{c}\text { Total } \\
\mathrm{n}=269 \\
(\text { No., \%) }\end{array}$ & $\begin{array}{c}\text { Control group } \\
\text { air leak }<5 \text { days } \\
\mathrm{n}=238(\text { No., } \%)\end{array}$ & $\begin{array}{c}\text { Air leak } \geq 5 \text { days } \\
\mathrm{n}=31 \text { (No., } \%)\end{array}$ & $P$-value ${ }^{*}$ \\
\hline \multicolumn{5}{|l|}{ Planned incision } \\
\hline VATS & $251(93.31)$ & $220(92.44)$ & $31(100)$ & \multirow{3}{*}{0.285} \\
\hline Thoracotomy & $16(5.95)$ & $16(6.72)$ & 0 & \\
\hline Sternotomy & $2(0.74)$ & 2() & 0 & \\
\hline \multicolumn{5}{|l|}{ Unplanned conversion } \\
\hline \multicolumn{5}{|l|}{ VATS to thoracotomy } \\
\hline Yes & $248(92.19)$ & $15(6.30)$ & $6(19.35)$ & \multirow{2}{*}{0.011} \\
\hline No & $21(7.81)$ & $223(93.70)$ & $25(80.65)$ & \\
\hline \multicolumn{5}{|l|}{ Lobectomy } \\
\hline Yes & $134(49.81)$ & $115(48.32)$ & $19(61.29)$ & \multirow{2}{*}{0.174} \\
\hline No & $135(50.19)$ & $123(51.68)$ & $12(38.71)$ & \\
\hline \multicolumn{5}{|l|}{ Lobectomy location } \\
\hline RUL & $42(15.61)$ & $31(13.03)$ & $11(35.48)$ & 0.001 \\
\hline RML & $10(3.72)$ & $10(4.20)$ & 0 & 0.611 \\
\hline RLL & $27(10.04)$ & $23(9.66)$ & $4(12.90)$ & 0.530 \\
\hline LUL & $33(12.27)$ & $30(12.61)$ & $3(9.68)$ & 0.778 \\
\hline LLL & $21(7.81)$ & $20(8.40)$ & $1(3.23)$ & 0.485 \\
\hline Bilobectomy & $9(3.35)$ & $8(3.36)$ & $1(3.23)$ & 0.969 \\
\hline \multicolumn{5}{|l|}{ Location bilobectomy } \\
\hline RUL/RML & $4(44.44)$ & $3(37.50)$ & $1(100)$ & \multirow{2}{*}{0.444} \\
\hline RML/RLL & $5(55.56)$ & $5(62.50)$ & 0 & \\
\hline Segmentectomy & $17(6.32)$ & $15(6.30)$ & $2(6.45)$ & 0.974 \\
\hline \multicolumn{5}{|l|}{ Location segmentectomy } \\
\hline LUL apical trisegment & $9(52.94)$ & $7(46.67)$ & $2(100)$ & \multirow{4}{*}{0.569} \\
\hline LUL lingula & $2(11.76)$ & $2(13.33)$ & 0 & \\
\hline LLL sup seg & $2(11.76)$ & $2(13.33)$ & 0 & \\
\hline RLL sup seg & $4(23.53)$ & $4(26.67)$ & 0 & \\
\hline Any upper lobectomy & $85(31.60)$ & $69(28.99)$ & $16(51.61)$ & 0.014 \\
\hline Any upper lobectomy or bilobectomy & $93(4.57)$ & $76(31.93)$ & $17(54.84)$ & 0.012 \\
\hline Wedge resection & $115(42.75)$ & $103(43.28)$ & $12(38.71)$ & 0.629 \\
\hline Adhesions $>30 \%$ pleural surface & $56(20.97)$ & $40(16.88)$ & $16(53.33)$ & $<0.001$ \\
\hline Incomplete major fissure & $140(92.72)$ & $119(91.54)$ & $21(100)$ & 0.384 \\
\hline \multicolumn{5}{|l|}{ Fissure division } \\
\hline Stapler & $103(72.54)$ & $86(71.07)$ & $17(80.95)$ & \multirow{3}{*}{0.744} \\
\hline Cautery & $6(4.23)$ & $6(4.96)$ & 0 & \\
\hline Combination & $33(23.24)$ & $29(23.97)$ & $4(10.05)$ & \\
\hline Intraoperative transfusion & $3(1.12)$ & $3(1.26)$ & 0 & 0.530 \\
\hline
\end{tabular}


Table 7. Logistic regression and multivariable regression analysis of surigical factors for study population of 269 consecutive pulmonary resections, October $1^{\text {st }} 2015$ to June $30^{\text {th }} 2016$.

\begin{tabular}{|c|c|c|c|c|}
\hline \multirow[t]{2}{*}{ Variable } & \multicolumn{2}{|c|}{ Univariable analysis } & \multicolumn{2}{|c|}{ Multivariable analysis } \\
\hline & Odds ratio $(95 \% \mathrm{CI})$ & $P$-value $*$ & Odds ratio $(95 \% \mathrm{CI})$ & $P$-value* \\
\hline $\begin{array}{l}\text { Unplanned conversion } \\
\text { VATS to thoracotomy }\end{array}$ & $3.57(1.3-10.0)$ & 0.016 & $3.37(1.2-9.6)$ & 0.023 \\
\hline Lobectomy & $0.99(0.8-1.3)$ & 0.951 & $1.78(0.8-4.0)$ & 0.170 \\
\hline \multicolumn{5}{|l|}{ Lobectomy location } \\
\hline RUL & $3.67(1.6-8.4)$ & 0.002 & $4.16(1.7-10)$ & 0.001 \\
\hline RML & - & - & - & - \\
\hline RLL & $1.38(0.4-4.3)$ & 0.574 & $1.27(0.4-4.1)$ & 0.692 \\
\hline LUL & $0.74(0.2-2.6)$ & 0.641 & $0.72(0.2-2.6)$ & 0.607 \\
\hline LLL & $0.36(0.04-2.8)$ & 0.332 & $0.37(0.04-2.9)$ & 0.349 \\
\hline Bilobectomy & $0.96(0.12-7.9)$ & 0.969 & $0.88(0.1-7.4)$ & 0.904 \\
\hline Segmentectomy & $1.02(0.2-4.7)$ & 0.974 & $1.04(0.2-4.8)$ & 0.962 \\
\hline Wedge resection & $0.82(0.4-1.8)$ & 0.629 & $0.81(0.4-1.9)$ & 0.632 \\
\hline $\begin{array}{l}\text { Adhesions }>30 \% \\
\text { pleural surface }\end{array}$ & $1.56(0.9-2.6)$ & 0.092 & $1.53(0.91-2.6)$ & 0.106 \\
\hline \multicolumn{5}{|l|}{ Fissure division } \\
\hline Stapler & - & - & - & - \\
\hline Cautery & $1.43(0.4-4.6)$ & 0.546 & $1.53(0.5-5.1)$ & 0.487 \\
\hline Combination & - & - & - & - \\
\hline
\end{tabular}

health-care costs [17]. The mean LOS for patients with PAL in our study was significantly longer at 12.6 days compared to 5.0 days for patients in the control group $(P<$ 0.0001). There was no association found between PAL and readmission rates at 30 and 90 days, or increased mortality.

Preoperative patient characteristics, validated scoring methods, and surgical factors were analyzed as possible risk factors. Normal BMI, compared to an overweight BMI, was a significant predictor of PAL. A relatively lower BMI has been found to be a risk factor in other studies [13] [14]. Brunelli et al. [13] developed a scoring system based assigning points based on individual risk factors, with a maximum score of 5.5, and includes 2 points for BMI $<25.5$. From the total score they assigned four risk categories which demonstrated increasing PAL incidence in their validation set. Rivera et al. [14] developed an Index of PAL (IPAL), in which BMI $<24$ increases IPAL, which is associated with increased risk of PAL. Patients with an overweight BMI have increased intra-abdominal pressure which forces the diaphragm cephalad helping to reduce the residual space following resection. There was a trend for age $>70$ to be associated with PAL which may be due to a more fragile lung parenchyma and reduced healing capacity in this population. There was also a trend for association of FEV1 $>80 \%$ and DLCO $>80 \%$ with decreased odds of PAL on multivariate analysis. This aligns with previous studies which have consistently found reduced pulmonary function due to 
Table 8. Logistic regression and multivariable regression analysis of patient characteristics for study population of 269 consecutive pulmonary resections, October $1^{\text {st }} 2015$ to June $30^{\text {th }} 2016$.

\begin{tabular}{|c|c|c|c|c|}
\hline \multirow[t]{2}{*}{ Variable } & \multicolumn{2}{|c|}{ Univariable analysis } & \multicolumn{2}{|c|}{ Multivariable analysis } \\
\hline & Odds ratio $(95 \% \mathrm{CI})$ & $P$-value & Odds ratio $(95 \% \mathrm{CI})$ & $P$-value* \\
\hline \multicolumn{5}{|l|}{ Age $($ years $)(n=269)$} \\
\hline $41-50$ & 1.00 (Reference) & - & 1.00 (Reference) & - \\
\hline $51-60$ & $1.31(0.4-4.8)$ & 0.681 & $1.22(0.3-4.5)$ & 0.766 \\
\hline $61-70$ & $0.79(0.2-3.0)$ & 0.728 & $0.74(0.2-2.8)$ & 0.662 \\
\hline $71-80$ & $2.21(0.7-7.4)$ & 0.198 & $1.92(0.6-6.6)$ & 0.300 \\
\hline$>80$ & $0.98(0.2-5.8)$ & 0.978 & $0.89(0.1-5.4)$ & 0.899 \\
\hline Age $>70$ years & $1.86(0.9-4.0)$ & 0.109 & $1.71(0.8-3.7)$ & 0.178 \\
\hline \multicolumn{5}{|l|}{$\operatorname{Sex}(n=269)$} \\
\hline Female & 1.00 (Reference) & - & 1.00 (Reference) & - \\
\hline Male & $1.08(0.5-2.3)$ & 0.831 & $1.30(0.6-2.9)$ & 0.509 \\
\hline \multicolumn{5}{|c|}{ Charlson co-morbidity score $(n=269)$} \\
\hline 0 & 1.00 (Reference) & - & 1.00 (Reference) & - \\
\hline $1-2$ & $1.24(0.5-2.9)$ & 0.621 & $1.05(0.4-2.6)$ & 0.922 \\
\hline $3-4$ & $1.65(0.5-5.0)$ & 0.375 & $1.26(0.4-4.2)$ & 0.705 \\
\hline$\geq 5$ & - & - & - & - \\
\hline \multicolumn{5}{|l|}{ BMI category: $(n=243)$} \\
\hline Underweight $(<18.5)$ & 1.00 (Reference) & - & 1.00 (Reference) & - \\
\hline Normal (18.5 - 25) & $3.16(1.3-7.5)$ & 0.009 & $3.28(1.3-8.0)$ & 0.009 \\
\hline Overweight (>25) & - & - & - & - \\
\hline \multicolumn{5}{|l|}{ FEV1 category $(\mathrm{n}=210)$} \\
\hline$<60 \%$ & 1.00 (Reference) & - & 1.00 (Reference) & - \\
\hline $60-80 \%$ & $0.40(0.1-1.8)$ & 0.237 & 0.34 & 0.176 \\
\hline$>80 \%$ & $0.57(0.1-2.2)$ & 0.416 & 0.38 & 0.210 \\
\hline \multicolumn{5}{|c|}{ FVC/FEV $1 \%$ category $(n=206)$} \\
\hline$<60 \%$ & 1.00 (Reference) & - & 1.00 (Reference) & - \\
\hline $60-80 \%$ & $1.07(0.3-4.0)$ & 0.918 & $0.79(0.2-3.1)$ & 0.739 \\
\hline$>80 \%$ & $0.69(0.2-3.2)$ & 0.639 & $0.48(0.1-2.4)$ & 0.377 \\
\hline \multicolumn{5}{|c|}{ DLCO $\%$ category $(n=202)$} \\
\hline$<60 \%$ & 1.00 (Reference) & - & 1.00 (Reference) & - \\
\hline $60-80 \%$ & $0.72(0.2-2.2)$ & 0.566 & $0.65(0.2-2.0)$ & 0.452 \\
\hline$>80 \%$ & $0.44(0.1-1.4)$ & 0.166 & $0.35(0.1-1.3)$ & 0.107 \\
\hline
\end{tabular}




\section{Continued}

\begin{tabular}{|c|c|c|c|c|}
\hline \multicolumn{5}{|c|}{ ECOG performance status category: $(n=266)$} \\
\hline ECOG 0 & 1.00 (Reference) & - & 1.00 (Reference) & - \\
\hline ECOG 1 & $1.04(0.4-2.5)$ & 0.920 & $1.18(0.5-2.9)$ & 0.725 \\
\hline ECOG 2 & $2.90(0.7-11.8)$ & 0.136 & $3.59(0.8-16.0)$ & 0.094 \\
\hline ECOG 3 & $2.18(0.2-20.6)$ & 0.498 & $2.51(0.3-24.3)$ & 0.428 \\
\hline \multicolumn{5}{|c|}{ ECOG grouping: $(n=266)$} \\
\hline ECOG 0-1 & 1.00 (Reference) & - & 1.00 (Reference) & - \\
\hline ECOG $\geq 2$ & $2.64(0.8-8.7)$ & 0.111 & $3.00(0.9-10.4)$ & 0.083 \\
\hline \multicolumn{5}{|c|}{ MRC dyspnea scores $(n=266)$} \\
\hline 1 & 1.00 (Reference) & - & 1.00 (Reference) & - \\
\hline 2 & 1.20 & 0.670 & $0.66(0.2-2.3)$ & 0.512 \\
\hline 3 & 2.14 & 0.272 & $1.01(0.2-6.0)$ & 0.993 \\
\hline 4 & - & - & - & - \\
\hline \multicolumn{5}{|c|}{ MRC dyspnea score grouping $(n=266)$} \\
\hline 1 & 1.00 (Reference) & - & 1.00 (Reference) & - \\
\hline$\geq 2$ & $1.25(0.6-2.7)$ & 0.571 & $0.81(0.2-2.6)$ & 0.729 \\
\hline
\end{tabular}

chronic obstructive pulmonary disease as a risk factor [5] [8] [9] [10] [11] [12]. There was also a trend for risk of air leak to differ based on primary diagnosis, however, due to the small sample size, we were unable to perform multivariate analysis.

The CCI was developed in 1987 by Charlson and colleagues in a cohort study of 559 medical patients as a weighted sum of patient comorbid conditions [20]. It was subsequently tested in a population of patients undergoing treatment for breast cancer, and when patients were grouped into CCI categories of $0,1-2,3-4$, and $\geq 5$, there was a stepwise increase in mortality with increasing CCI. Birim et al. [21] investigated using the CCI to predict surgical risk in patients operated on for non-small cell lung cancer, and found that the CCI was a strong predictor of major complications, but not minor complications which included air leak $>5$ days [21]. CCI grouped into the categories mentioned above was not found to be a predictor of PAL, however from univariate analysis of the individual Charlson comorbidities there was an association between cerebrovascular disease (CVD) and PAL, which may be due to smoking as a risk factor for CVD. For this study we investigated whether current smoking was a risk factor for PAL, and it is a limitation of this study that we did not take into consideration packyear smoking history. There was also a trend for association of uncomplicated diabetes mellitus with PAL which may be due to reduced healing capacity in diabetic patients. There is weak evidence of an association of ECOG $\geq 2$ with PAL and MRC dyspnea score did not have a significant association with PAL.

Surgical factors that had a statistically significant association with PAL include an unplanned conversion from VATS to thoracotomy, presence of adhesions, and right 
upper lobectomy. Conversion from VATS to thoracotomy occurred with operations in which it was difficult to access and resect necessary structures which resulted in increased lung manipulation. Some of the conversions from VATS to thoracotomy were performed due to the presence of dense adhesions. Previous studies have found adhesions to be a risk factor for PAL [5] [12] as their presence may cause tears in the lung parenchyma with manipulation of the lung, contributing to PAL. Right upper lobectomy involves both the major and minor fissures. There was a trend for association of lobectomy and upper lobectomy with PAL. Lobectomies involve resection of more lung parenchyma compared to sublobar resections, and combined with the effects of gravity, a larger residual apical space may prevent visceral and parietal pleural apposition for upper lobectomies.

Limitations include that this a single-centre study with a small sample size. Furthermore, as a retrospective observational study, the patient data collected and operation reports were not intended to be used for this purpose, and therefore there is bias introduced through the interpretation of these data sources.

\section{Conclusion}

In conclusion, unplanned conversion, adhesions, and right upper lobectomy were the most significant surgical factors associated with PAL in our study. We observed several trends consistent with previously described predictors in the literature; however, our study was limited by small sample size.

\section{References}

[1] Singhal, S., Ferraris, V.A., Bridges, C.R., Clough, E.R., Mitchell, J.D., Fernando, H.C., et al. (2010) Management of Alveolar Air Leaks after Pulmonary Resection. Annals of Thoracic Surgery, 89, 1327-1335. http://dx.doi.org/10.1016/j.athoracsur.2009.09.020

[2] Mueller, M.R. and Marzluf, B.A. (2014) The Anticipation and Management of Air Leaks and Residual Spaces Post Lung Resection. Journal of Thoracic Disease, 6, 271-284.

[3] Burt, B.M. and Shrager, J.B. (2014) Prevention and Management of Postoperative Air Leaks. Annals of Cardiothoracic Surgery, 3, 216-218.

[4] Stephan, F., Boucheseiche, S., Hollande, J., Flahault, A., Cheffi, A., Bazelly, B. and Bonnet, F. (2000) Pulmonary Complications Following Lung Resection. Chest, 118, 1263-1270. http://dx.doi.org/10.1378/chest.118.5.1263

[5] Brunelli, A., Montebverde, M., Borrin, A., Salati, M., Marasco, R. and Fianchini, A. (2004) Predictors of Prolonged Air Leak after Pulmonary Lobectomy. Annals of Thoracic Surgery, 77, 1205-1210. http://dx.doi.org/10.1016/j.athoracsur.2003.10.082

[6] Isowa, N., Hasegawa, S., Bando, T. and Wada, H. (2002) Preoperative Risk Factors for Prolonged Air Leak Following Lobectomy or Segmentectomy for Primary Lung Cancer. European Journal Cardio-Thoracic Surgery, 73, 1727-1731.

[7] Abholda, A., Liu, D., Brooks, A. and Burt, M. (1998) Prolonged Air Leak Following Radical Upper Lobectomy: An Analysis of Incidence and Possible Risk Factors. Chest, 113, 1507 1510. http://dx.doi.org/10.1378/chest.113.6.1507

[8] Liang, S., Ivanovic, J., Gilbert, S., Maziak, D.E., Shamji, F.M., Sundaresan, R.S., et al. (2013) Quantifying the Incidence and Impact of Postoperative Prolonged Alveolar Air Leak after 
Pulmonary Resection. Journal of Thoracic and Cardiovascular Surgery, 145, 948-954. http://dx.doi.org/10.1016/j.jtcvs.2012.08.044

[9] Bardell, T. and Petsikas, D. (2003) What Keeps Postpulmonary Resection Patients in Hospital? Canadian Respiratory Journal, 10, 86-89.

http://dx.doi.org/10.1155/2003/610570

[10] Cerfolio, R.J., Bass, C.S., Pask, A.H. and Katholi, C.R. (2002) Predictors and Treatment of Persistent Air Leaks. Annals of Thoracic Surgery, 73, 1727-1730. http://dx.doi.org/10.1016/S0003-4975(02)03531-2

[11] Stolz, A.J., Schutzner, J., Lischke, R., Simonek, J. and Pafko, P. (2005) Predictors of Prolonged Air Leak Following Pulmonary Lobectomy. European Journal of Cardio-Thoracic Surgery: Official Journal of the European Association for Cardio-Thoracic Surgery, 27, 334336. http://dx.doi.org/10.1016/j.ejcts.2004.11.004

[12] Lee, L., Hanley, S.C., Robineau, S.C., Sirois, C., Mulder, D.S. and Ferri, L.E. (2011) Estimating the Risk of Prolonged Air Leak after Pulmonary Resection Using a Simple Scoring System. Journal of the American College of Surgeons, 212, 1027-1032. http://dx.doi.org/10.1016/j.jamcollsurg.2011.03.010

[13] Brunelli, A., Varela, G., Refai, M., Jimenez, M.F., Pompili, C., Sabbatini, A. and Aranda, J.L. (2010) A Scoring System to Predict the Risk of Prolonged Air Leak after Lobectomy. Annals of Thoracic Surgery, 90, 204-209. http://dx.doi.org/10.1016/j.athoracsur.2010.02.054

[14] Rivera, C., Bernard, A., Falcoz, P.E., Thomas, P., Schmidt, A., Bénard, S., Vicaut, E. and Dahan, M. (2011) Characterization and Prediction of Prolonged Air Leak after Pulmonary Resection: A Nationwide Study Setting up the Index of Prolonged Air Leak. Annals of Thoracic Surgery, 92, 1062-1068. http://dx.doi.org/10.1016/j.athoracsur.2011.04.033

[15] DeCamp, M.M., Blackstone, E.H., Naunheim, K.S., et al. (2006) Patient and Surgical Factors Influencing Air Leak after Lung Volume Reduction Surgery: Lessons Learned from the National Emphysema Treatment Trial. Annals of Thoracic Surgery, 82, 197-207. http://dx.doi.org/10.1016/j.athoracsur.2006.02.050

[16] Irshad, K., Feldman, L.S., Chu, V.F., Dorval, J.-F., Baslaim, G. and Morin, J.E. (2002) Causes of Increased Length of Hospitalization on a General Thoracic Surgery Service: A Prospective Observational Study. Canadian Journal of Surgery, 45, 264-268.

[17] Varela, G., Jimenez, M.F., Novoa, N. and Aranda, J.L. (2005) Estimating Hospital Costs Attributable to Prolonged Air Leak in Pulmonary Lobectomy. European Journal of CardioThoracic Surgery, 27, 329-333. http://dx.doi.org/10.1016/j.ejcts.2004.11.005

[18] Burt, B.M. and Shrager, J.B. (2015) The Prevention and Management of Air Leaks Following Pulmonary Resection. Thoracic Surgery Clinics, 25, 411-419. http://dx.doi.org/10.1016/j.thorsurg.2015.07.002

[19] Merritt, R.E., Singhal, S. and Shrager, J.B. (2010) Evidence-Based Suggestions for Management of Air Leaks. Thoracic Surgery Clinics, 20, 435-448.

http://dx.doi.org/10.1016/j.thorsurg.2010.03.005

[20] Charlson, M.E., Pompei, P., Ales, K.L., et al. (1987) A New Method of Classifying Prognostic Comorbidity in Longitudinal Studies: Development and Validation. Journal of Chronic Diseases, 40, 373-383. http://dx.doi.org/10.1016/0021-9681(87)90171-8

[21] Birim, O., Maat, A.P., Kappetein, A.P., et al. (2003) Validation of the Charlson Comorbidity Index in Patients with Operated Primary Non-Small Cell Lung Cancer. European Journal of Cardio-Thoracic Surgery, 23, 30-34. http://dx.doi.org/10.1016/S1010-7940(02)00721-2

[22] Stenton, C. (2008) The MRC Breathlessness Scale. Occupational Medicine, 58, 226-227. 
http://dx.doi.org/10.1093/occmed/kqm162

[23] Oken, M.M., Creech, R.H., Tormey, D.C., et al. (1982) Toxicity and Response Criteria of the Eastern Cooperative Oncology Group. American Journal of Clinical Oncology, 5, 649655. http://dx.doi.org/10.1097/00000421-198212000-00014

Submit or recommend next manuscript to SCIRP and we will provide best service for you:

Accepting pre-submission inquiries through Email, Facebook, LinkedIn, Twitter, etc.

A wide selection of journals (inclusive of 9 subjects, more than 200 journals)

Providing 24-hour high-quality service

User-friendly online submission system

Fair and swift peer-review system

Efficient typesetting and proofreading procedure

Display of the result of downloads and visits, as well as the number of cited articles

Maximum dissemination of your research work

Submit your manuscript at: http://papersubmission.scirp.org/

Or contact ojts@scirp.org 